\title{
Correlation of Visual Prostate Symptom Score with International Prostate Symptom Score and Uroflowmetry Parameters in Nepalese Male Patients with Lower Urinary Tract Symptoms
}

\author{
Krishna Kaji Bhomi, ${ }^{1}$ Neeraj Subedi, ${ }^{1}$ Prem Prasad Panta ${ }^{2}$ \\ 'Department of Urology, Nepal Medical College, Jorpati, Kathmandu, Nepal, ${ }^{2}$ Department of Community Medicine, Nepal \\ Medical College, Jorpati, Kathmandu, Nepal.
}

\section{ABSTRACT}

Introduction: International prostate symptom score is a validated questionnaire used to evaluate the lower urinary tract symptoms in benign prostatic hyperplasia. Visual prostate symptom score is a new simplified symptom score with pictograms to evaluate the same. We evaluated the correlation of visual prostate symptom score with international prostate symptom score and uroflowmetry parameters in Nepalese male patients with lower urinary tract symptoms.

Methods: Male patients aged $\geq 40$ years attending the Urology clinic were enrolled in the study. They were given international prostate symptom score and visual prostate symptom score questionnaires to complete providing assistance whenever needed. Demographic data, examination findings and uroflowmetry parameters were noted. Correlation and regression analysis was used to identify correlation of the two scoring systems and uroflowmetry parameters.

Results: Among the 66 patients enrolled, only 10 (15.15\%) patients were able to understand English language. There was a statistically significant correlation between total visual prostate symptom score and international prostate symptom score $(\mathrm{r}=0.822 ; \mathrm{P}<0.01)$. The correlations between individual scores of the two scoring systems related to force of urinary stream, frequency, nocturia and quality of life were also statistically significant. There was also a statistically significant correlation of both scores with maximum flow rate and average flow rate.

Conclusions: There is a statistically significant correlation of visual prostate symptom score with international prostate symptom score and uroflowmetry parameters. IPSS can be replaced with simple VPSS in evaluation of lower urinary tract symptoms in elderly male patients.

Keywords: correlation; international prostate symptom score; lower urinary tract symptoms; uroflowmetry; visual prostate symptom score.

\section{INTRODUCTION}

The international prostate symptom score (IPSS) is a recommended symptom scoring instrument to be used for the baseline assessment of symptom severity in men presenting with lower urinary tract symptoms (LUTS). ${ }^{1}$
An important drawback of IPSS is that patients with lower educational level find difficulty completing the

Correspondence: Dr. Krishna Kaji Bhomi, Department of Urology, Nepal Medical College, Jorpati, Kathmandu, Nepal. Email: kkbhomi@hotmail.com, Phone: +977-9851070402. 
Bhomi et al. Correlation of Visual Prostate Symptom Score with International Prostate Symptom Score and Uroflowmetry ...

questionnaire. Studies have shown that $30-70 \%$ of men could not complete IPSS because they found the questions difficult to understand. ${ }^{2}$

To overcome the difficulties with IPSS, van der Walt et al developed a visual prostate symptom score (VPSS) which consist of pictograms to assess the LUTS. ${ }^{3}$ It has been found that VPSS correlates significantly with IPSS and could be completed without physician assistance by a greater proportion of men with limited education. VPSS has been compared with IPSS in African, Korean and Indonesian population. ${ }^{4-6}$

In this study we compared VPSS scores with IPSS scores and uroflowmetry parameters in Nepalese population with LUTS.

\section{METHODS}

This is a hospital based cross-sectional observational study conducted in Nepal Medical College Teaching Hospital over seven months period from May 2016 to November 2016. Ethical approval from Institutional Research/Ethical Sub-Committee was taken (approval number 51-072/073). Male patients aged $\geq 40$ years presenting in the Urology clinic with LUTS were enrolled in the study. Complete medical history was taken from each patient including literacy status, educational level, and language spoken. The patients were requested to complete IPSS questionnaire and then VPSS questionnaire. Whenever patient was unable to complete the questionnaire because of inability to understand the questionnaire, a medical person's assistance was offered.

Relevant physical examinations were done including evaluation of the prostate gland by digital rectal examination. Transabdominal ultrasonography was requested to evaluate size of the prostate and the post void residual urine (PVRU) volume. Uroflowmetry was conducted and maximum flow rate (Qmax), average flow rate (Qave) and voided volume were recorded. Sample size of 66 was calculated using the formula

$\mathrm{n}=\left\{\mathrm{Z}^{2}\left(1-\mathrm{r}^{2}\right) / \mathrm{d}^{2}\right\}+2$

Where error (d) $=0.13$; reliability coefficient at $95 \%$ confidence interval $(Z)=1.96$ and considering correlation coefficient $r=0.86$ from previous study by Heyns CF. ${ }^{7}$ Statistical analysis was done using SPSS version 16. The Pearson Product moment correlation coefficient was done to show the relationship between the variables and $P$ value was compared at 0.01 and 0.05 .

\section{RESULTS}

A total of 66 men were enrolled during the period from May 2016 to November 2016. IPSS and VPSS scores of the patients enrolled in this study are shown in Table 1. Mean age of the patients was $64.46 \pm 9.46$. Only $10(15.15 \%)$ patients were able to understand English language. Only seven $(10.60 \%)$ patients completed IPSS without assistance whereas 18 (27.27\%) patients completed VPSS without assistance.

\begin{tabular}{|lcc|}
\hline \multicolumn{2}{|l|}{ Table 1. Patient characteristics. } \\
\hline Parameters & IPSS & VPSS \\
Total & 17.45 & 8.83 \\
Weak stream & 3.24 & 3.19 \\
Frequency & 2.45 & 2.47 \\
Nocturia & 3.03 & 3.15 \\
QOL & 2.94 & 2.88 \\
\hline
\end{tabular}

Values are represented as mean. IPSS, International Prostate Symptom Score (total 35); QOL, Quality of life; VPSS, Visual Prostate Symptom Score (total 23).

\begin{tabular}{|c|c|c|}
\hline Correlation parameters & $\begin{array}{c}\text { Coefficient } \\
\text { (r) }\end{array}$ & $P$ value \\
\hline IPSS total Vs VPSS total & 0.822 & $<0.01$ \\
\hline $\begin{array}{l}\text { IPSS weak stream Vs VPSS } \\
\text { weak stream }\end{array}$ & 0.478 & $<0.01$ \\
\hline $\begin{array}{l}\text { IPSS frequency Vs VPSS } \\
\text { frequency }\end{array}$ & 0.41 & $<0.01$ \\
\hline $\begin{array}{l}\text { IPSS nocturia Vs VPSS } \\
\text { nocturia }\end{array}$ & 0.907 & $<0.01$ \\
\hline IPSS QOL Vs VPSS QOL & 0.762 & $<0.01$ \\
\hline IPSS Vs Qmax & -0.701 & $<0.01$ \\
\hline VPSS Vs Omax & -0.729 & $<0.01$ \\
\hline IPSS Vs Qave & -0.612 & $<0.01$ \\
\hline VPSS Vs Qave & -0.643 & $<0.01$ \\
\hline IPSS weak stream Vs Qmax & -0.321 & $<0.01$ \\
\hline VPSS weak stream Vs Qmax & -0.518 & $<0.01$ \\
\hline Age Vs Omax & -0.053 & 0.67 \\
\hline Age Vs PVRU & 0.043 & 0.73 \\
\hline
\end{tabular}

IPSS, International Prostate Symptom Score; QOL, Quality of life; VPSS, Visual Prostate Symptom Score, Qmax, Maximum flow rate; Qave, average flow rate; PVRU, post void residual urine.

There is a strong correlation between total IPSS and total VPSS scores with a correlation coefficient of $0.822(P<0.01)$ (Table 2, Figure 1). There is also a statistically significant correlation between individual IPSS and VPSS scores related to urinary stream, 
frequency, and nocturia. In addition, the quality of life (QOL) score assessed by IPSS and VPSS also showed a strong correlation with Pearson's correlation coefficient of $0.762(P<0.01)$.

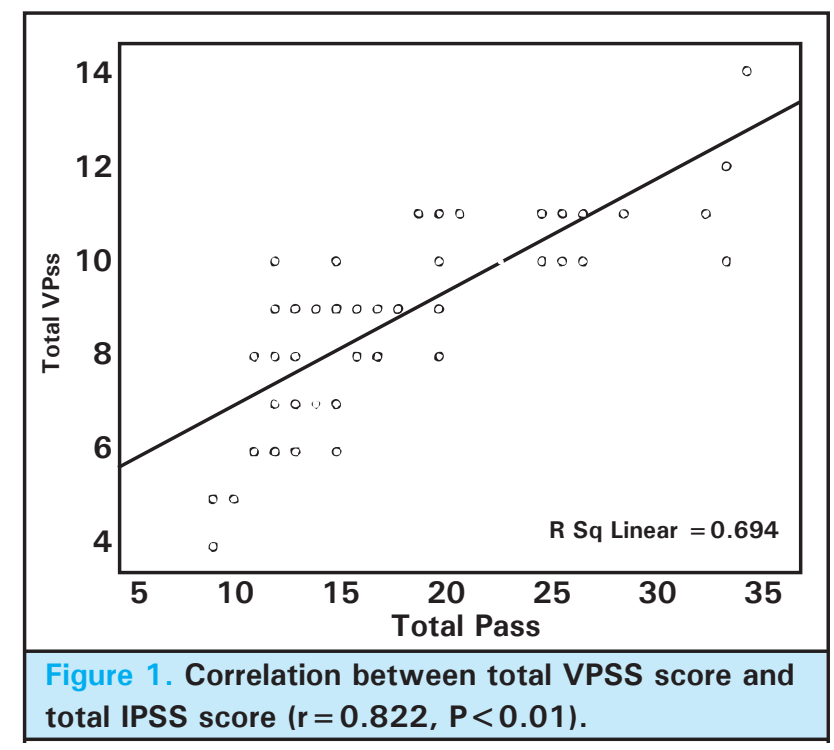

The IPSS and VPSS scores had statistically significant strong negative correlation with Qmax, with correlation coefficient of $-0.701(P<0.01)$ and $-0.729(P<0.01)$ respectively. Similarly there was also a significant negative correlation of IPSS and VPSS scores with Qave. In addition, IPSS and VPSS scores for weak stream also had significant correlation with Omax. There was no statistically significant correlation of age of the patient with Omax and PVRU.

\section{DISCUSSION}

The IPSS is currently a standard tool for the assessment of the severity of symptoms in men with LUTS. The American Urological Association guideline has recommended IPSS as a mandatory test in the evaluation and follow up of LUTS and benign prostatic hyperplasia $(\mathrm{BPH}) .^{8}$ It has been translated into more than 30 languages worldwide and studies have been done

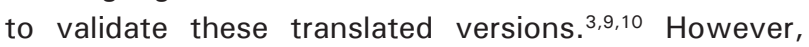
the complexity of IPSS causes problems for patients with low educational status who tend to misinterpret their scores, more often to a higher degree, possibly predisposing them to unnecessary intervention. ${ }^{11-13}$ Furthermore, the majority of men with $\mathrm{BPH}$ are aged 60 to 80 years and have visual and cognitive impairment. ${ }^{14}$ The small font size of IPSS questionnaire causes difficulty in completing the questionnaire for this group of patients.

Study done at Emory University (Atlanta, GA) revealed that for each symptom score question there was an inverse relationship between educational level and symptom misinterpretation. ${ }^{15}$ Patients with low educational status often need assistance to complete IPSS questionnaire. Using a medical personnel to assist the patient in completing IPSS introduces potential bias and imposes an additional burden on the medical staff. ${ }^{16,17}$

The VPSS includes schematic diagrams and can be understood easily even by illiterate elderly man. It reduces the risk of misinterpretation in translating the patient's symptoms into meaningful scoring system. Hence in developing country like Nepal where literacy levels in the elderly are low, replacing IPSS with VPSS to assess the severity of LUTS may greatly help to make appropriate decision for management of these patients.

Correlation between IPSS and VPSS was done initially by van der Walt et al in a teaching hospital serving a largely indigent population in the South Africa. ${ }^{9}$ They found that VPSS correlates significantly with IPSS in total score as well as individual parameters (weak stream, frequency and nocturia). Another study that enrolled 100 men with urethral stricture disease also demonstrated a correlation between total VPSS and total IPSS (correlation coefficient $r=0.845$, $\mathrm{P}<0.0001) .{ }^{18}$ A Namibian study that enrolled 100 men with LUTS showed a significant correlation between total IPSS and total VPSS scores (correlation coefficient $r=0.863, P<0.001) .{ }^{7}$ This correlation was stronger in groups with lower levels of education.

Our study also revealed a significant correlation between total IPSS and total VPSS scores with a correlation coefficient of $0.822(P<0.01)$. There is also a significant correlation between the individual parameters (weak stream, frequency and nocturia) of IPSS and VPSS. In addition IPSS QOL and VPSS QOL also revealed a strong correlation with correlation coefficient of $0.762(\mathrm{P}<0.01)$.

Uroflowmetry is a noninvasive test used in the evaluation of patients presenting with symptoms of bladder outlet obstruction (BOO). The Omax and the Qave are used in identifying patients with BOO. Studies have revealed correlation of VPSS and IPSS scores with Omax and Qave of the patients. ${ }^{3,19}$ This study also revealed a significant negative correlation of these parameters. In this study, the correlation of VPSS score with Omax ( $r=-0.729)$ and Qave $(r=-0.643)$ is somewhat stronger than the correlation of IPSS score with Qmax $(r=-0.701)$ and Qave $(r=-0.612)$. This indicates that VPSS is at least as reliable as IPSS and can be reliably used instead of IPSS to evaluate the subjective symptoms of LUTS which are related to objective uroflowmetry parameters.

Both IPSS and VPSS have not yet been validated in 
Nepali language, so we used English version of both the questionnaires. However only 10 (15.15\%) of our patients understood English language. This is why only seven $(10.60 \%)$ and $18(27.27 \%)$ patients completed IPSS and VPSS without assistance respectively.

Similarities between the findings of current study and those of earlier studies across the globe suggests that VPSS correlates well with IPSS and the uroflowmetry parameters regardless of cultural background.

\section{CONCLUSIONS}

Our study revealed a strong correlation of VPSS with IPSS and uroflowmetry parameters in evaluating the symptom severity in patients with LUTS. The total VPSS score as well as individual parameters along with quality of life score correlates significantly with their counterparts in IPSS. Hence VPSS can be used instead of complicated IPSS in a population with low literacy status.

\section{Conflict of Interest: None.}

\section{REFERENCES}

1. McNicholas TA, Kirby RS, Lepor H. Evaluation and nonsurgical management of benign prostatic hyperplasia. In: Wein AJ, editor. Campbell Walsh Urology $10^{\text {th }}$ ed. United States of America: Elsevier. 2010:2611-54. [Full Text]

2. Rodrigues Netto N Jr, de Lima ML, de Andrade EF. Latin American study on patient acceptance of the International Prostate Symptom Score (IPSS) in the evaluation of symptomatic benign prostatic hyperplasia. Urology. 1997;49(1):46-9. [PubMed | Full Text]

3. van der Walt CL, Heyns CF, Groeneveld AE, et al. Prospective comparison of a new visual prostate symptom score versus the international prostate symptom score in men with lower urinary tract symptoms. Urology. 2011;78:17-20. [PubMed | Full Text]

4. Park YW, Lee JH. Correlation between the visual prostate symptom score and international prostate symptom score in patients with lower urinary tract symptoms. Int Neurourol J. 2014;18:37-41. [PubMed | Full Text]

5. Heyns CF, van der Walt CL, Groeneveld AE. Correlation between a new visual prostate symptom score (VPSS) and uroflowmetry parameters in men with lower urinary tract symptoms. S Afr Med J. 2012;102:237-40. [PubMed]

6. Andika A, Yogi IG, Hari N. Comparison between visual prostate symptom score and international prostate symptom score in males older than 40 years in rural Indonesia. Prostate Int. 2014;2(4):176-81. [Full Text]

7. Heyns CF, Steenkamp BA, Chiswo J, et al. Evaluation of the visual prostate symptom score in a male population with great language diversity and limited education: a study from Namibia. S Afr Med J. 2014;104:353-7. [PubMed]

8. McVary KT, Roehrborn CG, Avins AL, et al. Update on AUA guideline on the management of benign prostatic hyperplasia. J Urol. 2011;185:1793-803. [PubMed]

9. Bailey A, Martin ML, Girman C. Development of a multiregional United States Spanish version of the International Prostate Symptom Score and the Benign Prostatic Hyperplasia impact index. J Urol. 2005;174:1896-1901. [PubMed]
10. Baida X, Garcia-Losa M, Dal-Re R. Ten-language translation and harmonization of the International Prostate Symptom Score: developing a methodology for multinational clinical trials. Eur Urol. 1997;31:129-40. [PubMed]

11. MacDiarmid SA, Goodson TC, Holmes TM, et al. An assessment of the comprehension of the American Urological Association Symptom Index. J Urol. 1998;159:873-4. [PubMed]

12. Cam K. BPH: how useful is a visual prostate symptom score for patients? Nat Rev Urol. 2011;8:536-7. [PubMed]

13. Netto Junior NR, de Lima ML. The influence of patient education level on the International Prostatic Symptom Score. J Urol. 1995;154:97-9. [PubMed]

14. Huh JS, Kim YJ, Kim SD. Prevalence of benign prostatic hyperplasia on Jeju Island: analysis from a cross-sectional community-based survey. World J Mens Health. 2012;30:131-7. [PubMed | Full Text]

15. Johnson TV, Abbasi A, Ehrlich SS, et al. Patient misunderstanding of the individual questions of the American Urological Association symptom score. J Urol. 2008;179:2291-4. [PubMed]

16. Plante M, Corcos J, Gregoire I. The international prostate symptom score: physician versus self-administration in the quantification of symptomatology. Urology. 1996;47:326-8. [PubMed]

17. Bozlu M, Doruk E, Akbay E. Effect of administration mode (patient vs physician) and patient's educational level on the Turkish version of the International Prostate Symptom Score. Int J Urol. 2002;9:417-21. [PubMed]

18. Wessels SG, Heyns CF. Prospective evaluation of a new visual prostate symptom score, the international prostate symptom score, and uroflowmetry in men with urethral stricture disease. Urology. 2014;83(1):220-4. [PubMed]

19. Ceylan Y, Gunlusoy B, Degrirmenci T, et al. Is new visual prostate symptom score useful as international prostate symptom score in the evaluation of men with lower urinary tract symptoms? A prospective comparision of 2 symptom scores in Turkish society. Urology. 2015;85:653-8. [PubMed] 